\title{
Screening of Differentiation-Specific Molecular Biomarkers for Colon Cancer
}

\author{
Lu Qia Yanqing Dinga,b \\ aDepartment of Pathology, School of Basic Medical Sciences, Southern Medical University, ${ }^{\text {bDepartment }}$ \\ of Pathology, Nanfang Hospital, Southern Medical University, China
}

\section{Key Words}

Colon cancer $•$ Differentiation grade $•$ Molecular marker $•$ Bioinformatics

\begin{abstract}
Background/Aims: Owing to the lack of effective molecular markers to evaluate colon cancer differentiation grade, screening of effective molecular markers for the diagnosis and treatment of colon cancer is of great significance. This study is a screening study for molecular markers related to the differentiation of colon using the tissue-specific genes of colon. Methods: This study compared the expression profiles of colon cancer at various differentiation grades and screened the down-regulated genes associated with decreased differentiation. IL22RA1 gene was derived from the intersection of obtained gene and colon tissue-specific genes. We used DriverDB and The Human Protein Atlas to analyze the expression level of IL22RA1 in various tissue cells, also used Kaplan-Meier method to analyze the correlation between IL22RA1 and the survival of colon cancer patients, and then used the ROC curve to analyze the specificity and sensitivity of IL22RA1 diagnosis of differentiated colon cancer. Results: We found that IL22RA1 gene expression was progressively down-regulated in high-differentiated, moderatedifferentiated, low-differentiated, and undifferentiated colon cancer tissues. Both RNA and protein levels of IL22RA1 were higher in colon tissues and colon cancer tissues than in other normal and cancer tissues. Comparison of IL22RA1 expression in different cancer cells found that IL22RA1 expression was significantly higher in CACO-2 colon cancer cells than in other cancer cells. Survival analysis showed that IL22RA1 gene expression was positively correlated with the overall survival rate of colon cancer patients $(P=0.0224)$. ROC curve analysis revealed that IL22RA1 expression had good specificity and sensitivity to stage II colon cancer. Conclusion: These findings suggest that IL22RA1 serves as a specific molecular marker for the differentiation of colon cancer.
\end{abstract}

\section{Introduction}

(C) 2018 The Author(s)

Published by S. Karger AG, Basel

The occurrence and metastasis of colon cancer remain detrimental to human health. Great differences exist with respect to speed of development and metastasis time of colon cancer in different patients [1]. Accurate diagnosis of benign or malignant tumors and determining the development speed is of great importance for the treatment and prognosis 
of patients with colon cancer. The degree of tumor differentiation signifies the difference between tumor and normal tissues. Highly-differentiated tumors closely resemble normal tissues, both morphologically and functionally, and are characterized by high maturity, lowgrade malignancy, slow progression and late metastasis. Conversely, low-differentiated tumors closely resemble a stem cell morphology, with high karyoplasmic ratio and weakness or loss of previous biological function. Hence, low-differentiated tumors are characterised by low maturity, high-grade malignancy, rapid progression, and early metastasis. The choice of regime in clinical treatment depends on tumour differentiation grade. According to histological characteristics, the differentiation grade of colon cancer includes highdifferentiation, moderate-differentiation, low-differentiation, and undifferentiation (grade I, grade II, grade III, and grade IV, respectively). Although some molecular markers have been used in clinical diagnosis and treatment of colon cancer, such as immunohistochemical labeling used in pathologic diagnosis [2], we have failed to identify specific molecular markers to evaluate the differentiation grade of colon cancer. This type of molecular marker would aid identification of the differentiation grade of colon cancer and determine the source of cancer tissue where morphological examination is unclear. Tissue differentiation grade is closely related to tissue-specific gene expression. Tissue-specific genes, also called luxury genes, are a group of genes whose function and expression are preferred in one or several tissues or cell types. Tissue-specific genes can code proteins that decide the growth of tissue cells and are associated with the biological functions of certain organs. This study aimed to screen the specific molecular markers involved in the differentiation of colon cancer, and to identify tissue-specific genes closely related with colon cancer differentiation. Finally, further study was performed to verify reliability of the selected genes and their diagnostic value in determining the differentiation grade of colon cancer.

\section{Materials and Methods}

\section{Screening of specific molecular markers for differentiation}

To identify molecular markers relevant to the differentiation grade of colon cancer, we screened colon tissue-specific genes that positively-correlated with various differentiation grades. After comparing differentially expressed genes in high-differentiation, moderate-differentiation, low-differentiation, and undifferentiation colon cancer cases, genes that were down-regulated with decreased differentiation were screened. The GSE2109 dataset (chip platform: Affymetrix Human Genome U133 Plus 2.0 Array [3]) retrieved from the Gene Expression Omnibus (GEO) database [4] includes 2158 cancer cases. From these cases, 239 cases of primary colon cancer were retrieved with details of tumor grade, including 8 cases of grade I (high differentiation), 176 cases of grade II(moderate differentiation), 51 cases of grade III (low differentiation) and 4 cases of grade IV (undifferentiation). Paired comparison was performed as follows: grade II vs. grade I, grade III vs. grade II, and grade IV vs. grade III. Genespring was used to screen differentially expressed genes. One-way analysis of variance (ANOVA) was performed for data analysis. P values were calculated with asymptotic and multiple testing corrections with the Bonferroni family-wise error rate (FWER)[5]. Differentially expressed genes with a P value less than 0.05 were selected with a single fold-change. The intersection was obtained from the selected differentially expressed genes according to descending order of differentiation grade. The intersection was obtained between the screened genes and the colon tissuespecific genes. Tissue-specific genes whose expression was down-regulated with decreased differentiation were screened as specific markers for differentiation. Tissue-specific genes of colon were obtained from two databases: Tissue-specific Gene Expression and Regulation (TiGER)[6], including 199 colon tissue-specific genes; and The Human Tissue-Specific Proteome [7] (belonging to The Human Protein Atlas project), including 165 colon tissue-specific genes. Based on the screening in this study, we identified IL22RA1 as a specific molecular marker for the differentiation of colon cancer.

Analysis of IL22RA1 expression

To determine IL22RA1 expression in colon tissues and colon cancer tissues, IL22RA1 expression data from the 239 colon cancer cases of GSE2109 was extracted. IL22RA1 expression in different grades was 


\section{Cellular Physiology Cell Physiol Biochem 2018;46:2543-2550 \begin{tabular}{l|l} 
and Biochemistry Published online: May 11, 2018 & $\begin{array}{l}\text { (c) } 2018 \text { The Author(s). Published by S. Karger AG, Basel } \\
\text { www.karger.com/cpb }\end{array}$
\end{tabular}

analyzed by one-way ANOVA. DriverDB[8] was used to analyze IL22RA1 expression in normal tissues and cancer tissues. The Human Protein Atlas (http://www.proteinatlas.org/) [9-11] was applied to analyze IL22RA1 protein expression in colon cancer tissues and other cancer cells.

Survival analysis of IL22RA1

IL22RA1 is a colon tissue-specific gene whose expression is down-regulated with decreased differentiation, therefore decreased IL22RA1 expression may promote decreased differentiation, increase the malignacy of colon cancer, and affect patient survival time. Hence, it is necessary to analyze the correlation between IL22RA1 expression and the survival time of colon cancer patients. The GSE39582 dataset retrieved from the GEO database was used to assess survival analysis of IL22RA1. This dataset includes 562 cases of colon cancer tissues with detailed overall survival time (chip platform: Affymetrix Human Genome U133 Plus 2.0 Array). IL22RA1 gene expression was retrieved from this data and ordered from low to high to represent low- and highexpression groups ( $\mathrm{n}=281)$, respectively. Kaplan-Meier [12] was used to compare survival rates of the two groups and the survival curve was obtained.

Receiver operating characteristic (ROC) curve analysis of IL22RA1

The ROC curve was used to verify specificity and sensitivity of the IL22RA1 gene for colon cancer differentiation grade. Based on the 239 colon cancer cases from the GSE2109 dataset, we found 176 grade II cases and 51 grade III cases. In this study, we extracted IL22RA1 gene expression data from these 227 cases and determined diagnostic efficacy of the IL22RA1 gene to grade II colon cancer through ROC curve analysis.

\section{Results}

Screening of specific molecular markers for differentiation

After comparing the expression profile of differentially expressed genes in colon cancer, 108

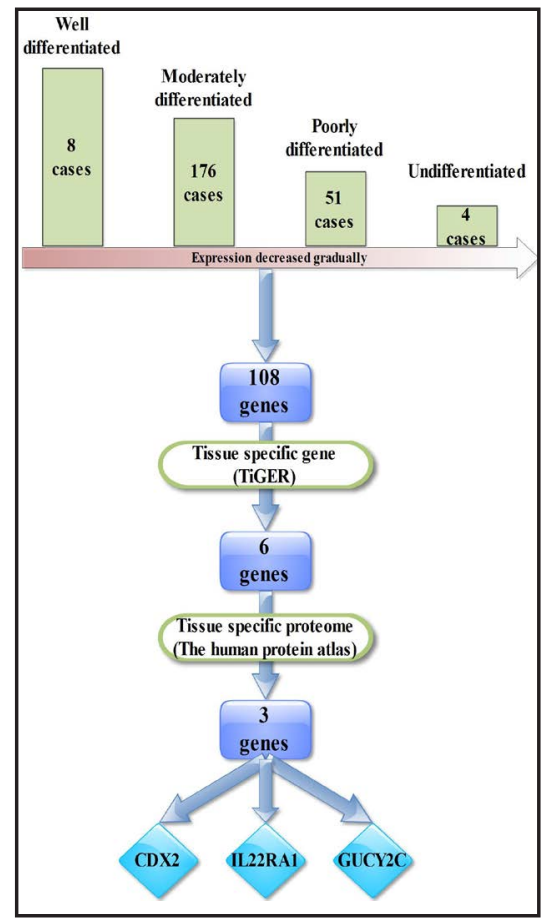

Fig. 1. Screening of specific molecular markers to identify colon cancer differentiation grade. genes were identified whose expression was down-regulated with decreased differentiation. The intersection was obtained between these 108 genes and 199 colon tissue-specific genes from the TiGER database, and six genes (CDX2, GUCY2C, IL22RA1, CFTR, SLC5A1, and TMC5) were selected. The intersection was further obtained from these selected 6 genes and 165 colon tissuespecific genes from the Human Tissue-Specific Proteome database, and three genes (CDX2, GUCY2C, and IL22RA1) were selected (Fig. 1). Previous studies have reported CDX2 and GUCY2C are differentiation-related genes in colon cancer. Hence, this study focused on the IL22RA1 gene.

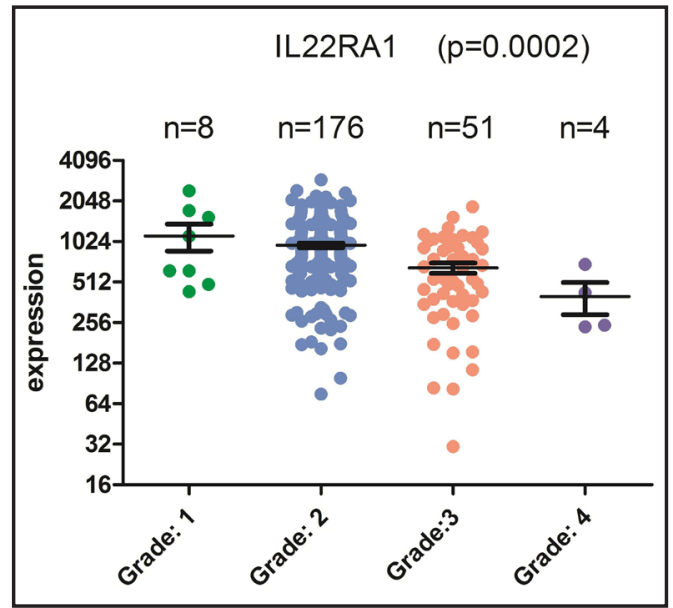

Fig. 2. IL22RA1 gene expression was downregulated with weakened differentiation grade. 


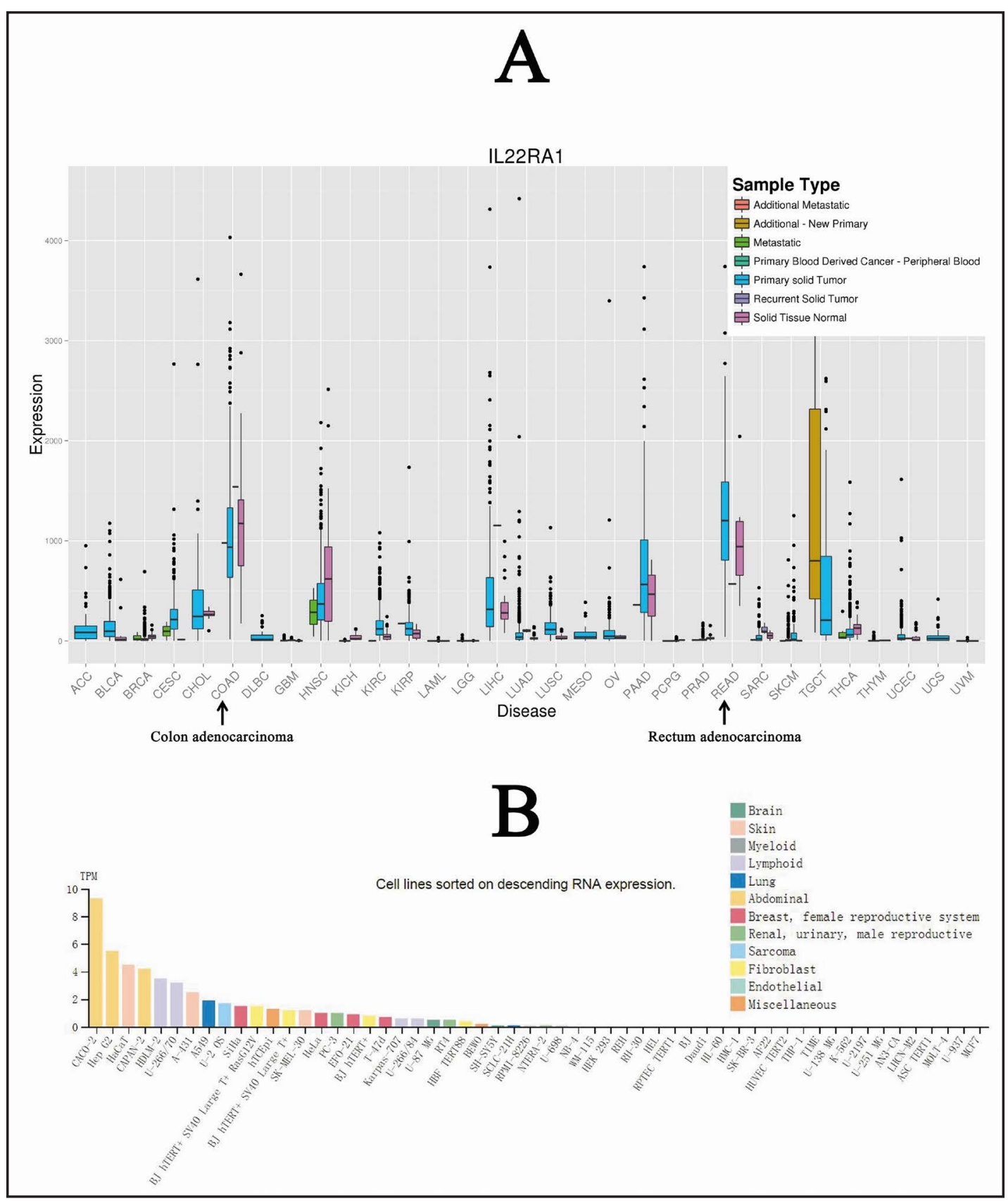

Fig. 3. A. IL22RA1 gene expression levels in normal and tumor tissues (data from DriverDB). COAD showed the RNA expression of colon and colon cancer. READ showed the RNA expression of rectum and rectal cancer. B. IL22RA1 gene expression level in cancer cells (data from The Human Protein Atlas).

\section{IL22RA1 expression}

IL22RA1 expression data of the 239 colon cancer cases were extracted from the GSE2109 dataset and drawn graphically (Fig. 2). IL22RA1 gene expression was different in colon cancer samples from different grades, with decreased expression in groups with lowdifferentiation. DriverDB analysis found that RNA levels of IL22RA1 gene were significantly higher in colon, colon cancer, rectum, and rectal cancer tissues than in other tissues (Fig. 3A). IL22RA1 protein level was detected immunohistochemically and analyzed by The Human Protein Atlas. The results showed that IL22RA1 protein level was higher in colon tissues compared with other normal tissues, and was higher in colon cancer tissue compared with KARGER 
other cancer tissues (Fig. 4). After analyzing IL22RA1 gene expression in cancer cells, we found that IL22RA1 gene expression was significantly higher in CACO-2 colon cancer cells than in other cancer cells (Fig. 3B). Analyses showed that both RNA and protein levels of IL22RA1 had obvious colon tissue-specificity and could serve as a promising molecular marker. IL22RA1 gene expression was down-regulated with decreased differentiation and might be a key gene to maintain normal differentiation of colon cancer.

\section{Survival analysis of IL22RA1}

Survival analysis showed that in the 562 cases of colon cancer, IL22RA1 gene expression was positively-correlated with overall pateint survival rate $(P=0.0224)$ (Fig. 5A). The IL22RA1 gene survival curve showed that survival rate was high in patients with high expression of IL22RA1, and low in patients with low expression of IL22RA1.

\section{Diagnostic efficacy of IL22RA1}

ROC curve analysis was performed on the IL22RA1 gene expression data in 227 cases of colon cancer (grade II and grade III) (AUC $=0.679, P=0$, 0001) (Fig. 5B). The results revealed that IL22RA1 gene expression had good specificity and sensitivity to grade II colon cancer and that IL22RA1 could serve as a valuable molecular marker for differentiation specificity in the diagnosis of colon cancer.

\section{Discussion}

This study compared colon cancer expression profile data in various differentiation grades and screened genes that were down-regulated with decreased differentiation. Colon-specific genes were then selected from these genes as the specific molecular markers of differentiation. After a further

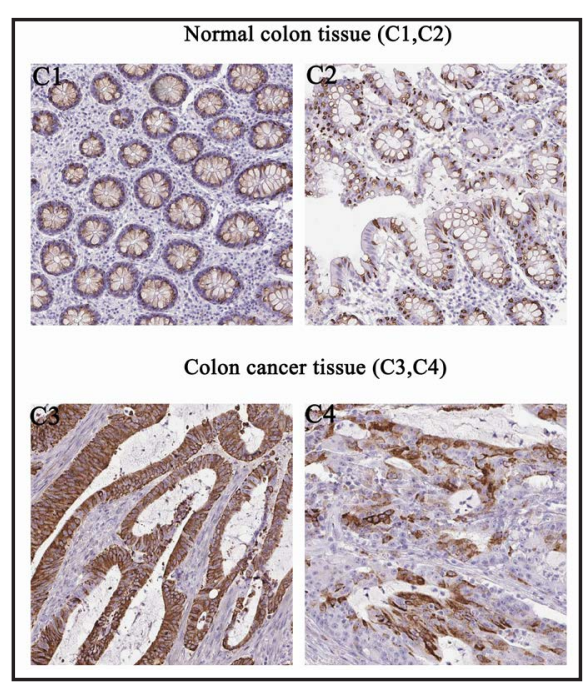

Fig. 4. IL22RA1 protein levels in normal and cancerous colon tissues (immunohistochemical staining, data from The Human Protein Atlas).

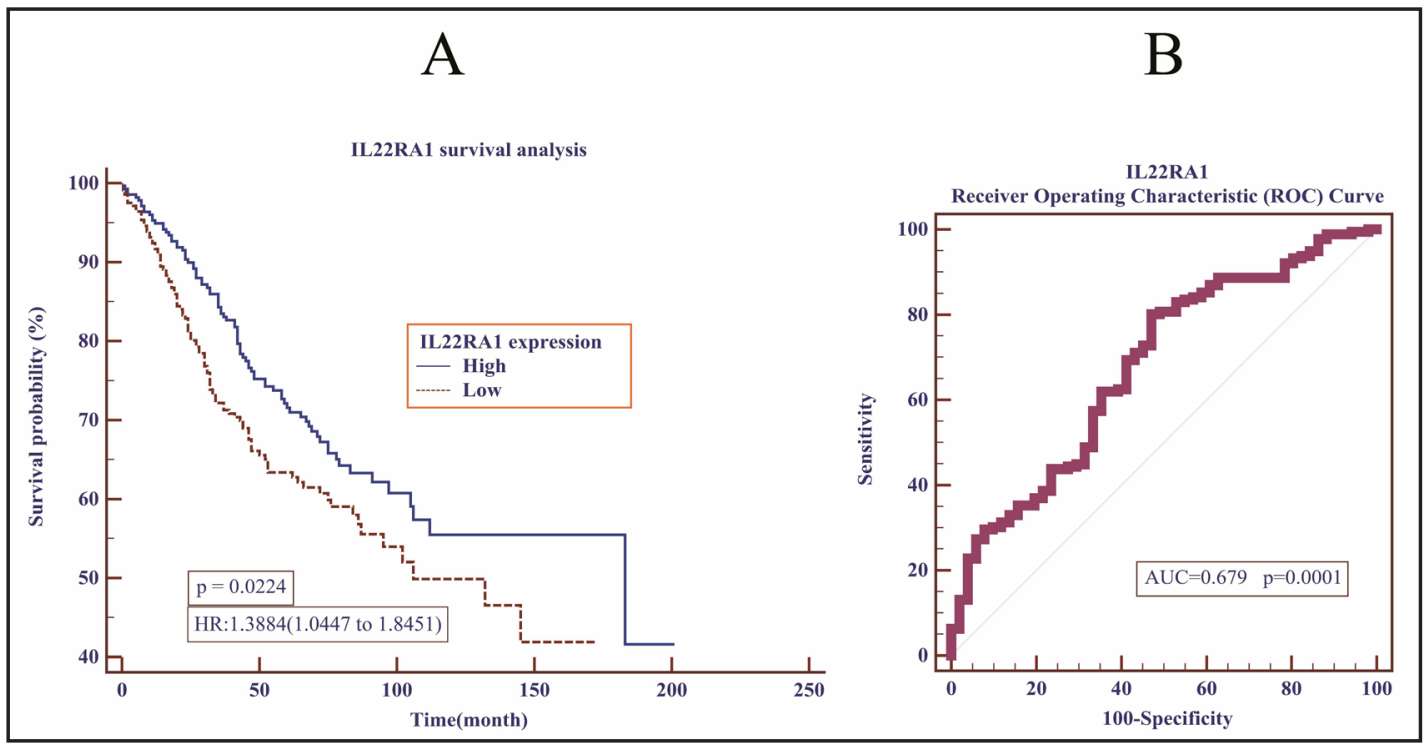

Fig. 5. A. IL22RA1 gene survival curve in patients with colon cancer. B. IL22RA1 gene ROC curve in colon cancer. 


\section{Cellular Physiology Cell Physiol Biochem 2018;46:2543-2550 \begin{tabular}{l|l|l} 
DOI: 10.1159/000489660 & $\begin{array}{l}\text { O 2018 The Author(s). Published by S. Karger AG, Basel } \\
\text { www.karger.com/cpb }\end{array}$
\end{tabular}

screening, three genes (CDX2, GUCY2C, and IL22RA1) were indicated. Previous studies have reported that CDX2 and GUCY2C are differentiation-related genes in colon cancer. CDX2 expression has been shown to inhibit the differentiation of intestinal neoplastic epithelium [13] and CDX2 gene polymorphism increase the risk of colon cancer [14]. Furthermore, deficiency of CDX2 expression is associated with poor prognosis of colon cancer $[15,16]$. CDX2 expression is specific in colon tissue and inhibits the migration of colon cancer cells [17]. These studies suggest that CDX2 can inhibit tumor growth [18].GUCY2C is also expressed specifically in colon tissue [19], and is often regarded as the molecular marker with highspecificity [20]. GUCY2C has been shown to inhibit the development and metastasis of colon cancer [21, 22], and has been used in the detection of colon cancer micrometastasis [23]. Furthermore, previous studies have reported that GUCY2C can regulate the balance between intestinal proliferation and differetiation [24] and is closely related to the recurrence and survival time of colon cancer [25]. Accumulated studies have shown the association between CDX2, GUCY2C, and colon cancer. Expression of both CDX2 and GUCY2C promotes the differentiation and inhibits the occurrence, development, and metastasis of colon cancer, and improves colon cancer prognosis as tumor suppressor genes [26]. Previous studies have reported that CDX2 and GUCY2C are differentiation-related genes in colon cancer, which implies reliability of the screening method used in this study. Hence, this study focused on analysis of the IL22RA1 gene.

This study reports an association of the IL22RA1 gene and cancer and found that IL22RA1 gene expression was downregulated with decreased differentiation. Both RNA and protein levels of IL22RA1 had obvious colon tissue-specificity. Interestingly, IL22RA1 gene expression was significantly higher in CACO-2 colon cancer cells compared with other cancer cells, and correlated with the overall survival time of colon cancer patients, survival curve indicates that IL22RA1 has a negative impact on the development of colon cancer, and suggests that IL22RA1 gene expression has an effect on the differentiation grade of colon cancer. Decreased IL22RA1 gene expression would therefore weaken colon cancer differentiation, resulting in low-differentiation or undifferentiation. As a result, patient survival time would be shortened because of promoted tumor cell proliferation, increased malignancy and early metastasis. ROC curve analysis revealed that IL22RA1 gene expression had good specificity and sensitivity for colon cancer. This study analyzed the close association between IL22RA1 and the differentiation grade of colon cancer by different methods. These results indicate IL22RA1 as a specific molecular marker for the differentiation of colon cancer. Detection of IL22RA1 gene expression therefore provides a new diagnostic marker for colon cancer.

\section{Acknowledgements}

This work was supported by the National Key Basic Research Program of China (973 Program, 2015CB554002), Project of the National Natural Science Foundation of china supported by NSFC-Guangdong Joint Fund (U1201226), the National Natural Science Foundation of China $(81472313,81773101)$.

\section{Disclosure Statement}

The authors declare to have no conflict of interests. 


\section{Cellular Physiology Cell Physiol Biochem 2018;46:2543-2550 \begin{tabular}{l|l} 
and Biochemistry Published online: May 11, 2018 & $\begin{array}{l}\text { (c) } 2018 \text { The Author(s). Published by S. Karger AG, Basel } \\
\text { www.karger.com/cpb }\end{array}$
\end{tabular} \\ Qi/Ding: Differentiation-Specific Molecular Biomarkers in Colon Cancer}

\section{References}

1 Cristobal A, van den Toorn HWP, van de Wetering M, Clevers H, Heck AJR, Mohammed S: Personalized Proteome Profiles of Healthy and Tumor Human Colon Organoids Reveal Both Individual Diversity and Basic Features of Colorectal Cancer. Cell Rep 2017;18:263-274.

2 Zlatian OM, Comanescu MV, Rosu AF, Rosu L, Cruce M, Gaman AE, Calina CD, Sfredel V: Histochemical and immunohistochemical evidence of tumor heterogeneity in colorectal cancer. Rom J Morphol Embryol 2015;56:175-181.

-3 Harbig J, Sprinkle R, Enkemann SA: A sequence-based identification of the genes detected by probesets on the Affymetrix U133 plus 2.0 array. Nucleic Acids Res 2005;33:e31.

4 Clough E, Barrett T: The Gene Expression Omnibus Database. Methods Mol Biol 2016;1418:93-110. Bland JM, Altman DG: Multiple significance tests: the Bonferroni method. BMJ 1995;310:170.

Liu X, Yu X, Zack DJ, Zhu H, Qian J: TiGER: a database for tissue-specific gene expression and regulation. BMC Bioinformatics 2008;9:271.

7 Uhlen M, Fagerberg L, Hallstrom BM, Lindskog C, Oksvold P, Mardinoglu A, Sivertsson A, Kampf C, Sjostedt E, Asplund A, Olsson I, Edlund K, Lundberg E, Navani S, Szigyarto CA, Odeberg J, Djureinovic D, Takanen JO, Hober S, Alm T, Edqvist PH, Berling H, Tegel H, Mulder J, Rockberg J, Nilsson P, Schwenk JM, Hamsten M, von Feilitzen K, Forsberg M, Persson L, Johansson F, Zwahlen M, von Heijne G, Nielsen J, Ponten F: Proteomics. Tissue-based map of the human proteome. Science 2015;347:1260419.

-8 Cheng WC, Chung IF, Chen CY, Sun HJ, Fen JJ, Tang WC, Chang TY, Wong TT, Wang HW: DriverDB: an exome sequencing database for cancer driver gene identification. Nucleic Acids Res 2014;42:D1048-1054.

-9 Lindskog C: The Human Protein Atlas - an important resource for basic and clinical research. Expert Rev Proteomics 2016;13:627-629.

10 Ponten F, Schwenk JM, Asplund A, Edqvist PH: The Human Protein Atlas as a proteomic resource for biomarker discovery. J Intern Med 2011;270:428-446.

-11 Ponten F, Jirstrom K, Uhlen M: The Human Protein Atlas--a tool for pathology. J Pathol 2008;216:387-393.

-12 Stel VS, Dekker FW, Tripepi G, Zoccali C, Jager KJ: Survival analysis I: the Kaplan-Meier method. Nephron Clin Pract 2011;119:c83-88.

13 Shang Y, Pan Q, Chen L, Ye J, Zhong X, Li X, Meng L, Guo J, Tian Y, He Y, Chen W, Peng Z, Wang R: Achaete scute-like 2 suppresses CDX2 expression and inhibits intestinal neoplastic epithelial cell differentiation. Oncotarget 2015;6:30993-31006.

14 Dai ZM, Fei YL, Zhang WG, Liu J, Cao XM, Qu QM, Li YC, Lin S, Wang M, Dai ZJ: Association of Vitamin D Receptor Cdx-2 Polymorphism With Cancer Risk: A Meta-Analysis. Medicine (Baltimore) 2015;94:e1370.

15 Bae JM, Lee TH, Cho NY, Kim TY, Kang GH: Loss of CDX2 expression is associated with poor prognosis in colorectal cancer patients. World J Gastroenterol 2015;21:1457-1467.

16 Hong KD, Lee D, Lee Y, Lee SI, Moon HY: Reduced CDX2 expression predicts poor overall survival in patients with colorectal cancer. Am Surg 2013;79:353-360.

17 Gross I, Duluc I, Benameur T, Calon A, Martin E, Brabletz T, Kedinger M, Domon-Dell C, Freund JN: The intestine-specific homeobox gene $\mathrm{Cdx} 2$ decreases mobility and antagonizes dissemination of colon cancer cells. Oncogene 2008;27:107-115.

$>18$ Hryniuk A, Grainger S, Savory JG, Lohnes D: Cdx1 and Cdx2 function as tumor suppressors. J Biol Chem 2014;289:33343-33354.

19 Waldman SA, Cagir B, Rakinic J, Fry RD, Goldstein SD, Isenberg G, Barber M, Biswas S, Minimo C, Palazzo J, Park PK, Weinberg D: Use of guanylyl cyclase C for detecting micrometastases in lymph nodes of patients with colon cancer. Dis Colon Rectum 1998;41:310-315.

20 Waldman SA, Barber M, Pearlman J, Park J, George R, Parkinson SJ: Heterogeneity of guanylyl cyclase C expressed by human colorectal cancer cell lines in vitro. Cancer Epidemiol Biomarkers Prev 1998;7:505514.

21 Li P, Schulz S, Bombonati A, Palazzo JP, Hyslop TM, Xu Y, Baran AA, Siracusa LD, Pitari GM, Waldman SA: Guanylyl cyclase $C$ suppresses intestinal tumorigenesis by restricting proliferation and maintaining genomic integrity. Gastroenterology 2007;133:599-607.

22 Lubbe WJ, Zuzga DS, Zhou Z, Fu W, Pelta-Heller J, Muschel RJ, Waldman SA, Pitari GM: Guanylyl cyclase C prevents colon cancer metastasis by regulating tumor epithelial cell matrix metalloproteinase- 9 Cancer Res 2009;69:3529-3536. 


\section{Cellular Physiology Cell Physiol Biochem 2018;46:2543-2550}

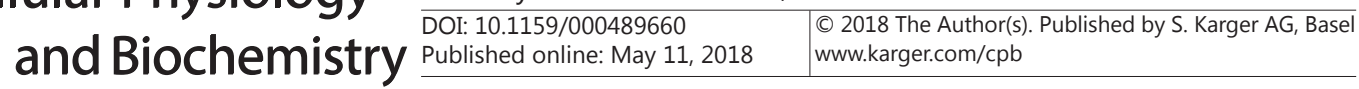

Qi/Ding: Differentiation-Specific Molecular Biomarkers in Colon Cancer

23 Frick GS, Pitari GM, Weinberg DS, Hyslop T, Schulz S, Waldman SA: Guanylyl cyclase C: a molecular marker for staging and postoperative surveillance of patients with colorectal cancer. Expert Rev Mol Diagn 2005;5:701713.

24 Pitari GM, Di Guglielmo MD, Park J, Schulz S, Waldman SA: Guanylyl cyclase C agonists regulate progression through the cell cycle of human colon carcinoma cells. Proc Natl Acad Sci U S A 2001;98:7846-7851.

25 Waldman SA, Hyslop T, Schulz S, Barkun A, Nielsen K, Haaf J, Bonaccorso C, Li Y, Weinberg DS: Association of GUCY2C expression in lymph nodes with time to recurrence and disease-free survival in pN0 colorectal cancer. JAMA 2009;301:745-752.

-26 Li P, Waldman SA: Corruption of homeostatic mechanisms in the guanylyl cyclase C signaling pathway underlying colorectal tumorigenesis. Cancer Biol Ther 2010;10:211-218. 